\title{
Rectosigmoid Inflammatory Cytomegalovirus Pseudotumor: A Disappearing Act
}

\author{
Arpan Patel ${ }^{1}$, Jihane N. Benhammou ${ }^{1}$, Gordon V. Ohning ${ }^{2}$, Michael Lewis ${ }^{3}$, David Ishimitsu ${ }^{4}$, \\ Joseph R. Pisegna ${ }^{2 *}$ \\ ${ }^{1}$ Division of Digestive Diseases, Department of Medicine, David Geffen School of Medicine at UCLA, CA \\ ${ }^{2}$ Division of Gastroenterology, Hepatology and Parenteral Nutrition, Department of Medicine VA Greater Los Angeles Healthcare System and \\ Department of Medicine David Geffen School of Medicine at UCLA, CA \\ ${ }^{3}$ Department of Pathology and Laboratory Medicine, VA Greater Los Angeles Healthcare System, CA \\ ${ }^{4}$ Department of Radiology VA Greater Los Angeles Healthcare System, CA
}

Received: October 10, 2015; Accepted: December 11, 2015; Published: December 28, 2015

*Corresponding author: Joseph R. Pisegna, Chief, Division of Gastroenterology, Hepatology and Parenteral Nutrition, VA Greater Los Angeles Healthcare System, Los Angeles, CA, 90073, Tel: +310-268-3578; Email: joseph.pisegna@va.gov

Keywords: Cytomegalovirus, Pseudotumor, Immunocompetent, Colitis, Rectosigmoid

\section{INTRODUCTION}

The gastrointestinal tract is a common site for cytomegalovirus (CMV) infections, especially in patients with underlying immunosuppression. We report a case of an elderly, immunocompetent patient in whom a rectosigmoid inflammatory polyp associated with CMV was identified in the evaluation of partial obstruction

\section{CASE REPORT}

The patient is an 89 year-old woman initially evaluated for five months of constipation described as slowed bowel movements associated with rectal pain, spasms, and tenesmus requiring frequent disimpaction and laxatives. She denied abdominal pain, hematochezia, or weight loss. Past medical history was positive for a history of a repaired rectocele, hysterectomy, and internal hemorrhoids. She denied any history of HIV, inflammatory bowel disease, solid organ or hematopoietic stem cell transplantation in the past.

Physical examination revealed an afebrile patient with a soft, non-tender abdomen. Rectal examination revealed poor anal tone, liquid stool, but no masses. The laboratory studies included a white blood count of 6700 cells $/ \mathrm{mm}^{3}$ (normal range 4500-11000), undetectable CMV titers, negative HIV-1 antibody, CD4 count 706 (normal range 464-1524) alkaline phosphatase $54 \mathrm{U} / \mathrm{L}$ (normal range 33-94), alanine aminotransferase $14 \mathrm{U} / \mathrm{L}$ (7.0-45.0), and aspartate aminotransferase $22 \mathrm{U} / \mathrm{L}$ (13-35).

Computerized tomography of the abdomen and pelvis (Fig. 1) revealed a rectum measuring $13 \mathrm{~cm}$ in diameter with mild wall thickening and fat stranding. Colonoscopy visualized a dilated rectum with a large, hard mass encompassing $75 \%$ of the mucosa, which was biopsied, but was impassable with an endoscope
(Fig 2 left panel). Pathological examination was reported as an inflammatory polyp with occasional inclusion bodies, with immunohistochemistry positive for CMV in multiple cells (Fig.3). A 2-week follow-up endoscopy revealed nodular mucosa at the region of the previously identified polyp but no organized mass (Fig. 2 right panel); pathology of the nodular mucosa revealed active chronic colitis with an inflammatory polyp, but with negative immunohistochemical stains for CMV.

\section{DISCUSSION}

Cytomegalovirus (CMV) is estimated to affect half of American adults with a seroprevelance of 40-100\% [1]. Primary infection and reactivation of $\mathrm{CMV}$ are most common in immunosuppressed patients [2].

Our case occured in the setting of constipation and a dilated rectum that resolved almost completely following two weeks of conservative therapy. Although CMV infection in immunocompetent hosts has been described, 34 of the 44 patients in a meta-analysis by Galiatsatos et. al, had comorbidities, such as renal disease, diabetes, and malignancy, which can weaken immune function [3] Our patient, who had none of the aforementioned medical problems and was not immunocompromised, complained mostly of constipation, in contrast to the typical symptoms of colitis.

Endoscopic evaluation of CMV colitis typically reveals shallow ulcerations of mucosa with colitis, though solitary mucosal ulcers, pseudomembrane formation, and ischemic colitis have been described [4]. CMV associated with pseudotumor development, however, has only been reported in eleven patients in the medical literature, with only three reported as being immunocompetent [5-9]. Two of these patients manifested typical symptoms of colitis whereas the third initially complained of right lower quadrant pain for 4 days. Pseudotumor locations included the cecum, ascending colon, and the sigmoid colon. In only one case was spontaneous disappearance of the tumor documented at the two week surveillance endoscopy whilst the two other patients 

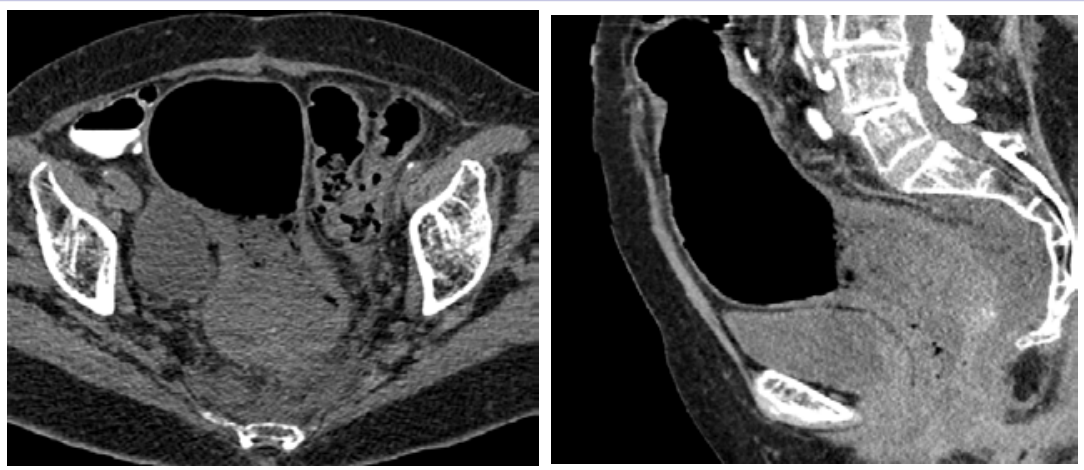

Figure 1: Computed tomography (CT) scan images of the abdomen and pelvis with A) axial images and B) sagittal images of a distended rectum with $13 \mathrm{~cm}$ diameter.
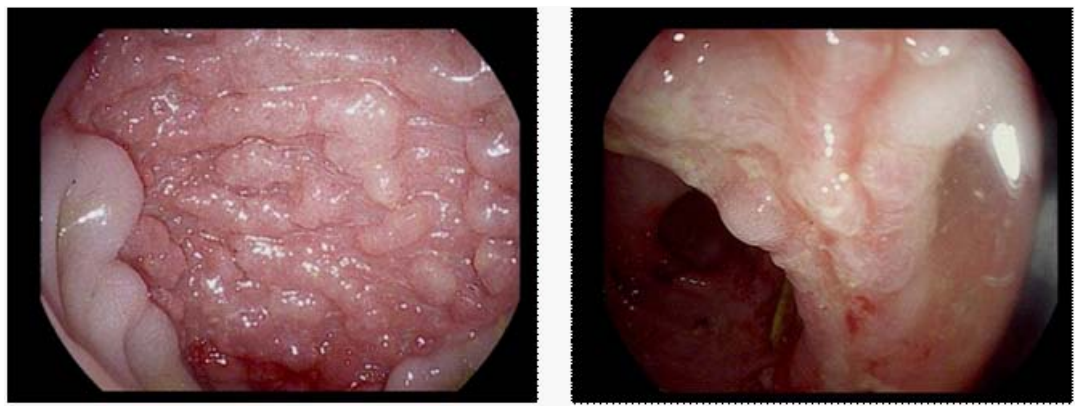

Figure 2: (Left panel) Endoscopic photograph of the nodular mass in the rectosigmoid colon, which was nearly circumferential and could not be passed by the smallest diameter endoscope available. (Right panel) Endoscopic photograph of the rectosigmoid colon showing nearly complete resolution of the mass. Endoscope was advanced without any resistance.
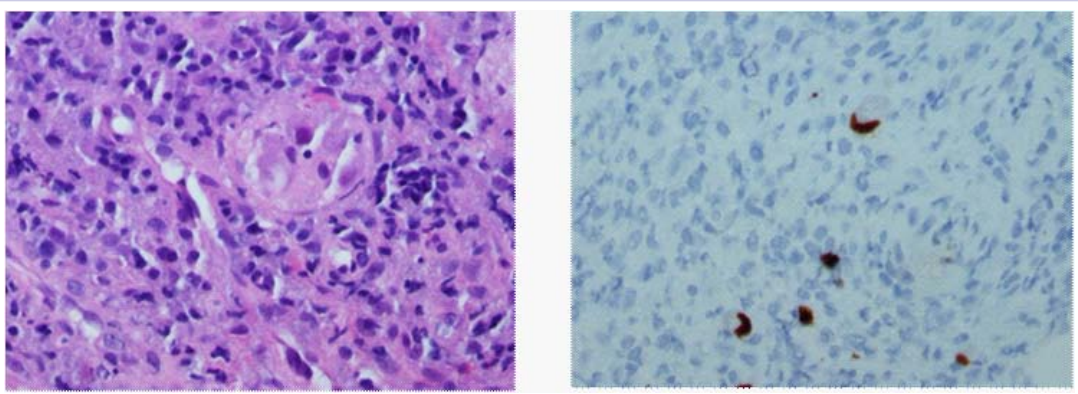

Figure 3: Histologic findings for biopsy of mass taken during endoscopy. A) Hematoxylin and eosin (H\&E) staining revealed inclusion bodies with a classic "owl's eyes" B) Immunohistochemical staining for CMV showed positive uptake in inclusion bodies.

were treated with surgery followed by ganciclovir $[8,9]$.

This is only the second case reported in the medical literature that describes an elderly, immunocompetent patient with a CMVassociated pseudotumor and the first case to describe a rectal CMV-related pseudotumor conservative treatment.

In elderly patients complaining of unexplained constipation, endoscopy should be considered if a diagnosis is not reached after history, physical examination, and imaging are completed. If a CMV pseudotumor is discovered, expectant management should be considered as an alternative to aggressive and potentially morbid antiviral therapy or surgery, though further experience and reporting of these cases is warranted.

\section{References}

1. Rahier, Ben-Horin S, Chowers Y, Conlon C, De Munter P, D'Haens G, et al. European evidence-based consensus on the prevention, diagnosis and management of opportunistic infections in inflammatory bowel disease. J Crohns Colitis. 2009;3(2):47-91. doi: 10.1016/j. crohns.2009.02.010.

2. Sissons JG, Carmichael AJ. Clinical aspects and management of cytomegalovirus infection. J Infect. 2002;44(2):78-83.

3. Galiatsatos, Shrier I, Lamoureux E, Szilagyi A. Meta-Analysis of 
Outcome of Cytomegalovirus Colitis in Immunocompetent Hosts. Dig Dis Sci. 2005;50(4):609-616

4. Kawasaki Osawa S, Sugimoto $\mathrm{K}$, Uotani $\mathrm{T}$, Nishino $\mathrm{M}$, Yamada $\mathrm{T}$, Sugimoto M, Furuta T, Ikuma M. "Cecal vanishing tumor associated with cytomegalovirus infection in an immunocompetent elderly adult. World J Gastroint Oncology. 2010 Nov 15; 2(11): 417-420. doi: 10.4251/wjgo.v2.i11. 417.

5. FalagasME, Griffiths J, Prekezes J, Worthington M. Cytomegalovirus colitis mimicking colon carcinoma in an HIV-negative patient with chronic renal failure.AM J Gastroenterol. 1996;91(1):168-169.

6. Rich JD, Crawford JM, Kazanjian SN, Kazanjian PH. Discrete gastrointestinal mass lesions caused by cytomegalovirus in patients with AIDS: report of three cases and review. Clin Infect Dis.
1992;15(4):609-614.

7. Imam S.Z, Khan A, Jaso JM, Foringer JR. Quiz page. CMV colitis presenting as a colonic mass. American journal of kidney diseases : the official journal of the National Kidney Foundation. 2010;55 (1) :A35-37.

8. Maiorana A, Torricelli P, Giusti F, Bellini N. Pseudoneoplastic appearance of cytomegalovirus-associated colitis in nonimmunocompromised patients: report of 2 cases. Clin Infect Dis. 2003;37(5):e68-71.

9. Kawasaki S, Osawa S, Sugimoto K, Uotani T, Nishino M, Yamada T, et al. Cecal vanishing tumor associated with cytomegalovirus infection in an immunocompetent elderly adult. World J Gastrointest Oncol. 2010; 2(11):417-20. doi: 10.4251/wjgo.v2.i11. 417. 